\title{
Planetary Protection Conventions for Landed Missions to Ocean Worlds
}

Brian G. Clement, Jet Propulsion Laboratory, California Institute of Technology, Pasadena, California

brian.g.clement@jpl.nasa.gov

(818) 354-3379

James N. Benardini, Jet Propulsion Laboratory, California Institute of Technology, Pasadena, California

Ryan C. Hendrickson, Jet Propulsion Laboratory, California Institute of Technology, Pasadena, California

Emily F. Klonicki, Jet Propulsion Laboratory, California Institute of Technology, Pasadena, California

Alvin L. Smith II, Jet Propulsion Laboratory, California Institute of Technology, Pasadena, California 


\section{Planetary Protection Conventions for Landed Missions to Ocean Worlds}

\section{Purpose}

Robotic missions to ocean worlds are among the most promising and exciting means for discovering extant or extinct life beyond Earth in the next few decades. Technologies that enable access to the ice and even liquid water on these bodies are rapidly evolving (Hendrix 2019). Indeed, the next decade will see multiple mission concepts for landing on and exploring ocean worlds continue to mature and/or launch. A key design driver for such missions is forward planetary protection, the practice of ensuring Earth (micro)organisms do not contaminate the target body. Ocean worlds, by definition, harbor liquid water and planetary protection (PP) requirements have traditionally prescribed the highest level of restrictions for direct water contact activities. Thus, landed ocean world missions present a novel challenge for demonstrating that water will not be either contacted or contaminated by Earth microorganisms. The former, which applies to landed missions only contacting icy surfaces, requires broad agreement on parameters (e.g. burial rates, distance to liquid water). The latter, applicable to subsurface exploration, requires ensuring sterilization of specific hardware and instruments in different mission phases. This white paper seeks to identify and recommend conventions that facilitate calculating the probability of biological contamination, and thereby enable ocean world mission concept development.

\section{Current NASA Requirements}

NASA's Planetary Protection Provisions for Robotic Extraterrestrial Missions (formerly NPR 8020.12D, currently NID 8020.109A) outlines requirements pertinent to ocean worlds, specifically seeking to limit forward contamination of bodies that are of "significant interest relative to the process of chemical evolution and/or the origin of life and for which scientific opinion provides a significant chance that contamination by spacecraft could compromise future investigations." Planetary protection requirements for target bodies reflect an approach originally used to allocate the risk of contaminating planetary bodies with viable Earth organisms to different countries. For Mars this probability of contamination was established by the international community then allocated to both the USSR and NASA over a 50-year period of exploration. After the first NASA implementation of this probabilistic requirement, Viking, standards were then adopted for bioburden cleanliness of the outbound spacecraft as a sole means of fulfilling the intent of the requirement given the large sensitivity of bioburden to, and increased knowledge of, the Mars environmental parameters in the risk analysis (Esposito 2000, Meltzer 2012). Currently, the ocean world PP requirement is still in a "Viking-like maturity" in terms of being able to define the input parameter models with higher fidelity and the associated spacecraft at launch bioburden. This Probability of Contamination ( $\left.P_{c}\right)$ limit, where contamination is defined as "the introduction of a single viable terrestrial microorganism into a liquid-water environment" is currently $10^{-4}$ (NID 8020.109A).

\section{How Planetary Protection Categories Apply to Ocean Worlds}

Currently, the restrictions for a specific body are considered to be given by the category listed in NID 8020.109A, with the cost and difficulty of meeting those requirements, and documenting compliance, increasing from I to V. The ocean worlds Europa and Enceladus fall 
under Category III and IV requirements; flyby/orbiter missions are Category III, while landed missions to both bodies are considered Category IV (most restricted). Ganymede, Titan, Triton, Pluto, and Charon are listed in the generally less restrictive Category II, but with a caveat that is telling for projects targeting unlisted bodies: missions visiting these water-harboring Category II bodies must also meet a $10^{-4}$ probability of contamination constraint. The Dawn flyby of Ceres is a good example; while Ceres missions are not categorized specifically by NPR 8020.12 (version C was in effect during planning), Dawn was assigned Category III (Rayman 2006). Thus, from a fundamental mission design perspective, the $\mathrm{P}_{\mathrm{C}}$ is applied to all missions exploring bodies that harbor water and chemistry consistent with the origin of biology as we currently understand it. Category V, involving restricted and unrestricted sample return to Earth, as it applies to ocean worlds has not been addressed by an active flight project.

\section{Calculating the Probability of Contamination}

For bodies with no known surface-subsurface transport, the $\mathrm{P}_{\mathrm{C}}$ may approach zero, but on bodies where transport to water is an active geological process, or mission goals include direct water access, the factors underlying the $P_{c}$ are critical for demonstrating compliance. The NPR directs that $P_{c}$ calculations include seven factors, at minimum (Table 1 ). While the probability of proliferation (Table $1, G$ ) is not currently well-constrained by our knowledge of ocean chemistry and therefore not useful for estimating $P_{c}$, the utility of the remaining six factors is dependent on mission structure. Flyby and orbiter missions can indeed utilize all six parameters, A-F, to meet the $P_{c}$. Landed missions will find that three of the seven are not useful, as the probability of landing safely (Table 1, D and E) is assumed to be near unity. Projects targeting subsurface water access are further restricted, as factor $F$ also trends towards unity because mission timescales are well within the lifespan of Earth microbes. This points to a key distinction between subsurface water access and landed missions - bioburden control is a clear focus for the former, but knowledge of (or agreed upon bounds on) geological processes can limit the importance of bioburden control for the latter. The Europa Clipper project has pioneered this approach.

Table 1. Factors for Calculating the Probability of Contamination per NPR 8020.12D (NID 8020.109A)

A. Bioburden at launch

B. Cruise survival for contaminating organisms

C. Organism survival in the radiation environment adjacent to the target

D. Probability of encountering/landing on the target, including spacecraft reliability

E. Probability of surviving landing/impact on the target

F. Mechanisms and timescales of transport to the subsurface

G. Organism survival and proliferation before, during, and after subsurface transfer

Note: Factors applicable to Landed Ocean Worlds missions are in bold, those applicable to

Subsurface Ocean Worlds missions are underlined. Those in italics apply primarily to flyby/orbiting missions, but are applicable to failure modes for landed missions during flight. 


\section{Recent Ocean Worlds Precedent: Europa Clipper}

Europa Clipper was designated a Category III PP mission as a Jupiter orbiter that would make tens of close passes by Europa. This designation requires the project to demonstrate $a<10^{-4} \mathrm{PC}_{\mathrm{C}}$ of an ocean or other liquid water body by a viable organism (NID 8020.109A). The Clipper mission developed a probability risk assessment (PRA) to satisfy this probability of contamination requirement. A 2018 PP workshop was used to confirm parameters for the PRA and resulted in a novel requirement approach to ocean world missions (McCoy et al., 2020 and Smith et al. 2020, both in preparation). Critical to this outcome was an agreement that the time interval considered for the $P_{C}$ calculation was limited to a 1000 -year Period of Biological Exploration (PBE). This new convention enabled the project to bound contamination using a conservative rate of resurfacing at Europa, assuming that contaminants could only get to liquid water if first "resurfaced" (subsumed) into the ice through surface turnover processes.

After the parameters enabling the PRA to satisfy the Pc requirement were reviewed and agreed upon, Mars-like bioburden requirements were also agreed to, in order to ensure reasonably clean assembly practices. Clipper's subsequent bioburden requirements are subtly different, in that the project is limiting areal densities of bacterial spores instead of limiting the total number of bacterial spores at launch, as is the convention for Mars requirements. The Clipper spore density requirements are either $<300$ or $<1,000 \mathrm{spore} / \mathrm{m}^{2}$, with one of the two values established based on characteristics of each hardware element. Components were given a bioburden density limit of $<1,000$ spore $/ \mathrm{m}^{2}$ if they met at least one of the following conditions:

- Unable to withstand a 3-log HMR (i.e. $110^{\circ} \mathrm{C}$ for 20 hours)

- Cannot be cleaned (via standard alcohol cleaning)

- Expected to reach 2.5 Mrad by first impact (2.5 Mrad being a sterilizing dose)

- Would be sterilized by impact heating $\left(500^{\circ} \mathrm{C}\right.$ for 0.5 seconds)

Hardware that did not meet at least one of these conditions were given a bioburden limit of $<300$ spores $/ \mathrm{m}^{2}$. Such requirements serve to contain the overall survival chances of organisms should an extremely unlikely event (or combination of events) occur; simply having fewer microbial passengers limits the likelihood of that organisms capable of proliferation on a specific target survive to a liquid water body.

Clipper is the first mission to utilize this unique arrangement of probability of contamination and bioburden requirements. This unique approach may serve as a template for future ocean world missions when PRAs demonstrate the probability of contaminating a water body is sufficiently low.

\section{Enabling Future Ocean Worlds Missions}

The PBE serves as an example of the types of conventions that allow future ocean world flight projects to design hardware elements and concepts of operation that facilitate PP compliance. It is both conservative, assuming scientific exploration will be completed at Europa in the next 1000 years, and enabling, setting a boundary that is amenable to calculation. Establishing such conventions for key parameters such as geological turnover rate and ice shell thickness will further guide future mission concepts. It should be noted that the recommended bounds are distinct from means or likely ranges used in science investigations in that they 
would be conservative and designed to encompass all likely values, explicitly for planning Planetary protection compliance and calculating a mission's Pc. As an example, statements supporting such parameters might take the form "there is no evidence suggesting a rate outside the range of $X-Y$ ". The specific conventions critical to protecting ocean worlds directly relate to mission elements, both hardware and operations.

Understanding and further developing peer-reviewed models for key end-to-end environmental parameters for organism survival in the radiation environment and subsurface transport rates are critical in enabling landed missions to ocean worlds. This would allow the science community to have a more standardized approach to probabilistic risk assessments to help balance the intent of the requirement so that future missions will have a clear path as to the approach so that missions would not have to construct and invest in full fledge PRAs in parallel with bioburden mitigation measures.

\section{Surface (Lander) Planetary Protection Elements for Ocean World Missions}

Surface landed missions would include those missions designed to survey and sample from a one or more surface locations; examples include the Johns Hopkins University Applied Physics Laboratory-led Dragonfly mission to Titan and the Europa Lander mission concept led by Jet Propulsion Laboratory. Like Clipper, these missions must demonstrate a Pc below $10^{-4}$, which could be accomplished through a PRA alone or in combination with robust bioburden mitigation. Key elements for the PRA include: (i) a demonstrably reliable flight system, capable of flying to the target with a low probability of failures that lead to unintended impacts, (ii) well-supported bounds on the rates at which geological processes subsume landed (or impacted) elements into the ice and transport those elements into contact with liquid water and (iii) an understanding of the lethality factors any launched bioburden would encounter.

Bioburden mitigation steps can clearly play a role but, as was shown by the Clipper PRA, operate like a step function in the $P_{C}$ calculation, only producing significant reductions when the probability of a viable organism on the entire landed system falls significantly below 1 . The Europa Lander Mission Concept embraced this by designing in elements that permit pre-launch microbial reduction on the surfaces of all landed and impacted elements after encapsulation in a protective "biobarrier" and terminal sterilization systems that utilize heat to sterilize electronics in the instrument and avionics vaults at the end of mission. It's worth noting that prior to the 1000-year PBE convention, the longevity of Earth microbes required such stringency for a Europa mission - the death of dormant-but-viable organisms able to survive to a flight to Europa cannot be reliably estimated and, therefore, the period of concern for surface turnover and transport to liquid would be in the millions of years. When similar arguments can be made for other ocean worlds, the PBE may result in landed missions needing limited bioburden mitigation.

\section{Planetary Protection Elements for Sub-surface Access Missions to Ocean Worlds}

Unlike surface-focused missions, subsurface access missions would deliver significant instrument, mobility, and communication systems directly into a crevasse or water phase. Fortuitously, a primary requirement for a subsurface mobility platforms such as probes and autonomous robotic architectures - the ability to exclude high pressure liquid water- facilitates bioburden reduction by ensuring that external treatments do not easily compromise internal components typically incompatible with microbial reduction processes such as power, payload, 
computing and navigation elements. In fact, those missions gaining subsurface access via melting and or drilling would create and descend in an essentially continuous water layer through the development of a melt jacket that would require sterility on all water contacting surfaces from the beginning of their descent operations. Moreover, the mission design would need to ensure that non-sterile, non-water contact elements (e.g. electronics, internal mechanisms) were either sterilized at the end of mission or protected from water contact for 1000 years. Sterility and/or water exclusion would need to be demonstrated to a high level of probability in each phase of the mission. As an example, a subsurface mobility platform at Europa would require sterile external surfaces during descent, sterile fluid-handling pathways (inflow and outflow) during science and water-jetting system operations and either a robust water-excluding shell able to resist all foreseeable ambient pressures for 1000 years or terminal sterilization systems to render all internal components sterile at end of mission. Such a platform may take advantage of in-flight microbial reduction from environmental or engineered sterilization processes. All of these technologies are currently feasible or under development but, under current conventions, sterilization remains difficult to integrate into the PC calculation. Conceptually, sterilization indicates a state can be reached at which all Earth microbes associated with a hardware element are demonstrably dead, yet this binary, live/dead assumption is not amenable to calculating the PC, opening another area in which clear conventions are needed to enable ocean world missions

\section{Recommended Conventions to Establish}

\section{Geological boundary conditions}

Recommendation: Develop a series of conventions, specifically for planetary protection use, that define geological boundary conditions at each ocean world critical to estimating the transit time from surface to liquid water.

Currently, any group proposing exploration of an ocean world must collect, analyze, and establish the boundaries for such parameters as resurfacing rate, ice shell thickness, and shallowest depth of water, prior to designing a mission concept compatible with Planetary Protection. Due to the nature of ocean worlds science, such findings will be debatable and subject to change because an early-phase mission concept activity rarely has the wherewithal to engage in workshops or reviews specifically for Planetary Protection. Instead, mission concepts often establish a robust PP compliance strategy only after key mission architecture trades have been completed. Developing the recommended conventions for each ocean world as a NASA-funded activity would facilitate mission proposals that have PP compliance included in the initial design, saving cost and time relative to developing workarounds in later phases.

\section{Sterilization}

Recommendation: Establish a convention for estimating the probability that one or more organisms (i.e. probability of a non-sterile state) survive exposure to a sterilizing lethality factor.

The single most direct means of meeting the $P_{c}$ requirement is to sterilize all flight elements visiting the target prior to launch. Unfortunately, sterilizing a spacecraft would require engineering compromises and development costs likely to reduce the number and scope of missions and reduce scientific output. Instead, missions to ocean worlds may adopt a just-in- 
time approach to sterilization where needed. The leading example of this is the terminal sterilization systems included in the Europa Lander mission concept that would heat and destroy sensitive electronics (which inevitably contain non-sterile, off-the-shelf components).

The heat sterilization target, $\geq 500^{\circ} \mathrm{C}$ for $\geq 0.5$, is the current convention for assuming sterility; it is not data-derived and all temperatures below this are, also by convention, limited to 6-log reduction of bacterial spores. Notably, this conservatism is driven by the PP-specific need to target a broad range of poorly characterized organisms. In contrast, the biomedical industry typically defines sterility by heat as a doubling of the treatment that reliably kills $10^{6}$ of the most resistant disease-causing organisms. Unlike the PP version of sterilization, this practice is amenable to calculating the probability that one or more organisms survived the treatment. Current PP conventions for other microbial reduction modalities, notably particle radiation and certain applications of vapor-phase hydrogen peroxide (VHP), also include sterilization as an endpoint, but not as a probability. Designing ocean world missions that utilize microbial reduction modalities before launch or in-transit, either as a defined treatment capability or calculated from an environmental exposure, would be simplified by conventions for estimating the probability of sterility.

\section{Integrating Molecular Biological Methods}

\section{Recommendation: Establish conventions for incorporating biomolecular indicators of bioburden on spacecraft hardware into the $\mathbf{P}_{\mathrm{c}}$ calculation.}

Genetics can eclipse the need for broad sterilization by revealing which organisms are actually associated with the spacecraft hardware, from assembly to launch. This information can expand the toolkit of classical cultivation-based microbial verification for enumeration, phylogenetic identification and high-resolution characterization of microbial traits and biochemical capabilities in order to define organisms that survive each mission phase (launch, cruise, landing and surface operations). Indeed, studies utilizing DNA have been undertaken to analyze microbes present in spacecraft assembly environments over the past decade, and are beginning to be used on samples collected from flight hardware on missions such as Mars 2020. These data often come in the form of organism types thought to be present based on DNA sequences recovered, but often yield little information on the absolute abundance of any one organism or the sampled population. Therefore, fractional lethality (e.g. an $\mathrm{N}$-log reduction due to particle radiation) is difficult to calculate. A convention for incorporating this information into the $P_{c}$ calculation might include specific methods for bounding the total bioburden quantity and the fractional abundance of specific organisms or types as well as means of calculating their survival at different times over the mission duration. This would greatly benefit missions that transit through and/or visit regions with high lethality factors (e.g. Jovian radiation) by allowing them to calculate environmental lethality for only the organisms actually present on the spacecraft in their $\mathrm{P}_{\mathrm{C}}$ calculation, further validating and establishing an ocean worlds, or target body-specific, pre-launch bioburden cleanliness requirement.

\section{Discussion and Summary}

Ocean world missions will be a key focus of future robotic exploration of the Solar System and concepts for both surface and subsurface investigations will evolve towards full-fledged flight projects in the next decade. Providing a means by which PP compliance strategies may be 
readily designed will enable mission concept development. We suggest here that PP conventions be established for each ocean world as specific products for mission designers. Such conventions are not novel - instead of $P_{c}$ calculations, missions to Mars have long used spore burden limits and impact calculations out to only 50 years after launch (well below the survival time of many bacterial spores). Current PP conventions for ocean world missions include the 1000-year PBE utilized by the Europa Clipper mission and the Mars-like bioburden limits that ensure a reasonably clean spacecraft. Additional conventions suggested here include geological parameters related to the rate at which water is contacted by landed elements, expanded use of sterilization probability and methods for integrating organism identity, and type into Pc calculations.

\section{References}

Esposito L. et al., (2000) Preventing the Forward Contamination of Europa, National Research Council, Washington, DC.

Hendrix, A. R., T. A. Hurford, L. M. Barge, M. T. Bland, J. S. Bowman, W. Brinckerhoff, B. J. Buratti, M. L. Cable, J. Castillo-Rogez, G. C. Collins, S. Diniega, C. R. German, A. G. Hayes, T. Hoehler, S. Hosseini, C. J. A. Howett, A. S. McEwen, C. D. Neish, M. Neveu, T. A. Nordheim, G. W. Patterson, D. A. Patthoff, C. Phillips, A. Rhoden, B. E. Schmidt, K. N. Singer, J. M. Soderblom, and S. D. Vance, (2019) Astrobiology, 19(1) 1-27, http://doi.org/10.1089/ast.2018.1955

Meltzer, M. (2011), When Biospheres Collide: A History of NASA's Planetary Protection Programs, NASA, Washington, DC.

NASA Interim Directive (NID) 8020.109A (2017) Planetary Protection Provisions for Robotic Extraterrestrial Missions, https://nodis3.gsfc.nasa.gov/OPD_docs/NID_8020_109A_.pdf

Rayman, M. et al., (2006) Dawn: A mission in development for exploration of main belt asteroids Vesta and Ceres, Acta Astronautica, 58(11) 605-616, doi:10.1016/j.actaastro.2006.01.014.

\section{Acknowledgement}

This work was carried out at the Jet Propulsion Laboratory, California Institute of Technology, under a contract with the National Aeronautics and Space Administration (80NM0018D0004). 